\title{
SEGMENTACIÓN DE MERCADO DE ACUERDO A ESTILOS DE VIDA DE CONSUMIDORES DE VINO ORGÁNICO DE LA REGIÓN METROPOLITANA DE CHILE
}

\author{
MARKET SEGMENTATION ACCORDING TO LIFESTYLES OF ORGANIC WINE \\ CUSTOMERS IN THE METROPOLITAN REGION OF CHILE
}

\author{
Marcos Germán Mora González; Nicolás Santiago Magner Pulgar ${ }^{1}$; Ricardo Marchant Silva ${ }^{1}$
}

\begin{abstract}
RESUMEN
El desarrollo de la vitivinicultura orgánica en Chile responde al desarrollo de una demanda potencial, asociada a la seguridad alimentaria y conservación medioambiental, lo que la transforma en una actividad promisoria, que se inserta en una industria vitivinícola globalizada y exitosa, pero que al mismo tiempo enfrenta desafíos referentes a conocer las demandas de los consumidores nacionales e internacionales. En este sentido, la siguiente investigación determina estilos de vida de consumidores de vino de la Región Metropolitana, y de acuerdo a esto segmenta el mercado, encontrando tres grupos de consumidores cada uno denominado "Sociables e indiferentes hacia el vino orgánico" segmento equivalente al 40,32\% del mercado; "Actitud positiva hacia el vino orgánico" segmento equivalente al 22,58\% del mercado y "Consumidores de productos orgánicos" segmento equivalente al $37,10 \%$.

Palabras clave: Vino orgánico, estudio de mercado, segmentación, estilos de vida.
\end{abstract}

\begin{abstract}
The development of organic winemaking in Chile responds to the development of a potential demand, linked to food security and environmental conservation, which transforms it into a promising activity, which is part of a global and successful wine industry, but at the same time facing challenges relating to meet the demands of consumers nationwide and abroad. In this regard, the following investigation determines lifestyles of consumers who came from the metropolitan area, and according to this market segments, finding three groups of consumers each called, "Social and indifferent toward organic wine" segment equivalent to $40.32 \%$ of the market; "Positive attitude toward organic wine" segment equivalent to $22.58 \%$ of the market; and "Consumers of organic products" segment equivalent to $37.10 \%$.
\end{abstract}

Key words: Organic wine, market research, segmentation, lifestyles.

\section{INTRODUCCIÓN}

La aparición en el mercado de un grupo importante de consumidores comprometidos con el medio ambiente ha determinado que una proporción de empresas estén cambiando sus prácticas habituales de producción por otras más responsables con su entorno (Fraj et al., 2004), dando paso a productos reconocidos como orgánicos o ecológicos, los que, aunque presentan una participación reducida en el consumo de alimentos, son considerados un nicho de mercado con gran potencial de crecimiento. Tal es el caso de Estados Unidos, en donde las ventas de productos orgánicos alcanzaron en el año 2003 los 10,4 billones de dólares, con tasas de crecimiento de $20,4 \%$ en los últimos años, atrayendo la atención de investigadores, empresas de alimentos y departamentos de agricultura. (Gifford y Bernard, 2006; Gentile y Rodríguez, 2005).

Es así como la comunidad científica ha desarrollado múltiples estudios de mercado, con el objeto de establecer los factores que intervienen en el comportamiento del consumidor de productos orgánicos, precisando que existen al menos tres grupos de variables, a saber: a) sociodemográficas; b) actitudes hacia el alimento orgánico, y c) estilos de

1 Universidad de Chile, Departamento de Economía Agraria. Av. Santa Rosa 11315, La Pintana - Santiago. Chile. Casilla 1004. Santiago.e-mail: mmorag@uchile.cl,nmagner@uchile.cl, ricardo.marchant@vtr.net. 
vida. Respecto al primer grupo, se ha observado que no existe una relación consistente en su utilización para explicar el comportamiento del consumidor orgánico $\mathrm{y}$, aunque permite resolver algunas cuestiones puntuales, no servirían para identificar adecuadamente un segmento de mercado (Kassarjian, 1971; Laroche et al., 2001), dando paso al análisis de actitudes hacia un alimento orgánico y estilos de vida, de forma que garantice la comprensión de este tipo de conducta (Bigné, 1997; Laroche et al., 2001; Fraj y Martínez, 2002).

Dentro de la temática de la investigación de mercados alimentarios, específicamente para vino y otros alimentos orgánicos, desde principios de los noventa destacan los trabajos en el sur de Australia para vinos, Lockshin et al. (2006) en vinos en Australia, Martínez-Carrasco et al. (2006); Gil y Sánchez (1997), Bernabéu et al. (2004) y Mora (2004) en España para vino de calidad, Brugarolas et al. (2004) en productos ecológicos en España, Howard y Allen (2006) y Gifford y Bernard (2006) en productos orgánicos en Estados Unidos.

\section{MATERIALES Y MÉTODOS}

La hipótesis del presente trabajo plantea que es posible determinar segmentos en el mercado chileno de vino orgánico a partir de sus estilos de vida. Para esto se realizó una encuesta entre los meses de octubre y diciembre de 2007 a consumidores de vino de la comuna de La Florida, escogiendo ésta por ser la tercera comuna con el mayor número de habitantes, presentar la mayor homogeneidad en la distribución del ingreso y una gran cantidad de plazas de venta, lo que la hace particularmente representativa de la realidad nacional.

El muestreo fue aplicado a un universo de 365.563 habitantes de la comuna, utilizando una hipótesis de normalidad para poblaciones finitas, considerando un nivel de confianza de $95 \%$ ( $\mathrm{K}=$ $1,96)$, y un error de $4,8 \%$, para extraer una muestra de 420 encuestas.

La encuesta incluyó una breve presentación del equipo investigador, objetivos de la investigación y el cuestionario, el cual estuvo conformado por un cien por ciento de preguntas cerradas relativas a: variables descriptivas de consumo, actitudes del consumidor hacia el vino orgánico, variables de estilo de vida, intención de compra hacia nueve prototipos de vino orgánico y variables sociodemográficas.
El método estadístico utilizado consistió en la aplicación de análisis factorial de componentes principales para determinar actitudes hacia el vino orgánico y estilos de vida de los encuestados, análisis de conglomerados jerárquicos utilizando el método de Ward para segmentar el mercado. Los segmentos fueron descritos mediante análisis de varianzas (ANDEVA) para variables continuas, y tablas de contingencia utilizando el estadístico chi cuadrado en el caso de variables discretas, lo que permite inferir si dos o más magnitudes de frecuencias de casos de la población pueden ser consideradas similares (Schnettler et al., 2007).

Finalmente se realizó un análisis conjunto para determinar preferencias hacia atributos representativos del vino, como lo son variedad de uva vinificada, precio, viña y denominación de origen (Luque, 2000; Malhotra, 1997). El análisis conjunto es una técnica multivariante que se utiliza específicamente para entender cómo los encuestados desarrollan preferencias hacia un producto o servicio real o hipotético, combinando cantidades separadas de valor que le proporciona cada atributo, siendo la utilidad la base conceptual que permite medir el valor en el análisis conjunto. (Hair et al., 1999). El producto de análisis correspondió a vino orgánico combinando cuatro atributos, los cuales fueron: variedad vinificada, precio, viña productora y denominación de origen, las que son utilizadas en diversas investigaciones de esta naturaleza (Johnson et al., 1991; Gil y Sánchez, 1997; Sánchez y Gil, 1997; Bernabéu et al., 2001; Martínez Carrasco, 2002; Barroso et al., 2004; Mora y Espinoza, 2005 y Mora et al., 2006). En relación al diseño del análisis, se establecieron niveles para cada uno de los cuatro atributos, siendo estos variedad vinificada (Ensamble; Carmenère; Cabernet sauvignon), precio por botella de $750 \mathrm{cc}(\$ 2.250$; $\$ 2.500 ; \$ 2.750$ ), viña productora (Concha y Toro; Ventisquero), y denominación de origen (Maule, Colchagua, Maipo). Finalmente, con la finalidad de disminuir el número de combinaciones generadas (estímulos), que en total son $36(3 \times 2 \times 3 \times 2)$ y de esta forma realizar una adecuada recopilación de información, se extrajeron nueve estímulos o prototipos de productos mediante un contraste ortogonal. Los prototipos fueron identificados con letras de la A hasta la I: A) vino orgánico, ensamble, $\$ 2.250$, viña Concha y Toro, D.O. Valle del Maule. B) vino orgánico, ensamble, $\$ 2.750$, viña Concha 
y Toro, D.O. Valle de Colchagua. C) vino orgánico, carmenère, \$2.500, viña Concha y Toro, D.O. Valle de Colchagua. D) vino orgánico, ensamble, \$2.500, viña Ventisquero, D.O. Valle del Maipo. E) vino orgánico, cabernet sauvignon, $\$ 2.750$, viña Concha y Toro, D.O. Valle del Maipo. F) vino orgánico, carmenère, \$2.250, viña Concha y Toro, D.O. Valle del Maipo. G) vino orgánico, cabernet sauvignon, $\$ 2.250$, viña Ventisquero, D.O. Valle de Colchagua. H) vino orgánico, cabernet sauvignon, \$2.500, viña Concha y Toro, D.O. Valle del Maule. I) vino orgánico, carmenère, \$2.750, viña Ventisquero, D.O. Valle del Maule.

\section{RESULTADOS Y DISCUSIÓN}

La frecuencia de consumo de vino predominante fue semanal con un $39,76 \%$, seguido por ocasional con un $33,03 \%$ y mensual con un $19,57 \%$. En relación a la cantidad consumida en cada ocasión, el 51,07\% declaró que consumía entre una a tres copas, mientras que los consumos de menos de una copa y de media a una botella presentaron un 20,02 y $22,32 \%$ respectivamente. La plaza de compra de mayor preferencia fue el supermercado con un $72,17 \%$, seguida con un $20,49 \%$ por la botillería, mientras que las compras realizadas por Internet, en tiendas especializadas y directamente en la viña, alcanzaron el 7,34\% restante. Del total de consumidores de vino, el 20,49\% declaró ser consumidor de vino orgánico. Del 79,21\% restante, que expresó no haber consumido vino orgánico, un 58,30\% declaró no conocer ese producto. En relación a las personas encuestadas que declararon no consumir vino, se estimó que el $27,23 \%$ no consume porque le desagrada su sabor, un 19,90\% no consume bebidas alcohólicas y un $18,32 \%$ no consume porque prefiere la cerveza, mientras que el $16,23 \%$ restante no consumía por diversos motivos (salud incompatible, religión, alto precio y alto grado alcohólico).

\section{DETERMINACIÓN DE ESTILOS DE VIDA}

Por medio del análisis factorial de componentes principales con método de rotación de normalización varimax con Kaiser, se determinaron tres estilos de vida, obteniendo una medida de adecuación muestral de Kaiser-Meyer-Olkin. (KMO) de 0,639.
Factor 1: Estilo de vida dinámico y natural. Este factor explica un $25,42 \%$ del total de varianza. Presenta una correlación positiva con las siguientes afirmaciones: "Procuro consumir alimentos bajos en grasa" $(0,858)$, "Me preocupo por mi salud" $(0,822)$, "Tengo con frecuencia comidas de trabajo" $(0,568)$ y "Hago deporte regularmente" $(0,526)$. Este factor presentó correlación negativa con la afirmación "Suelo consumir a menudo comida rápida" $(-0,748)$. Este estilo de vida fue denominado "Dinámico y natural" pues agrupa afirmaciones que hacen referencia a un estilo de vida dedicado al trabajo complementado con actividades que mantienen un ritmo dinámico y natural. Kavak y Gumusluoglu (2006), en una investigación de mercado realizada en Turquía sobre la relación que tienen el etnocentrismo y los estilos de vida en la disposición de compra de 2.000 encuestados, determinaron que existía un estilo de vida que denominaron "preocupados por la salud", los cuales presentaban rasgos muy similares, pues presentó correlación positiva con preferir alimentos bajos en calorías y usar alimentos dietéticos una vez al día. Además, y asociado al consumo de alimentos orgánicos, Kafka y Alvensleben (1998) señalan que en el mercado de Dinamarca los consumidores de productos orgánicos presentan un claro estilo de vida preocupado por la salud.

Factor 2: Estilo de vida sociable y lúdica. Este factor explica el 18,13\% del total de varianza. Presenta una correlación positiva hacia las afirmaciones "Mi tiempo libre lo dedico a viajar" $(0,808)$ y "Tengo con frecuencia reuniones con amigos" $(0,785)$. El segundo factor fue denominado estilo de vida "Sociable y lúdica" pues los consumidores asocian en forma positiva al factor actividades relacionadas con el esparcimiento y las relaciones sociales con su entorno. Fraj et al. (2004) determinaron estilos de vida asociados a consumidores de productos ecológicos, dentro de los cuales uno de ellos, particularmente el denominado espíritu aventurero, presenta similitudes pues está correlacionado positivamente con afirmaciones como "me gusta hacer cosas nuevas y emocionantes". Además, al igual que el factor anterior, estos resultados confluyen con los resultados encontrados en el mercado turco, pues en esa oportunidad se detectó un estilo de vida denominado "extrovertido", el cual presentó correlación positiva con afirmaciones como gusto 
de las fiestas con amigos y me considero el alma de mi hogar (Kavak y Gumusluoglu, 2006).

Factor 3: Estilo de vida de cultura y buena mesa. Este factor explica el 17,51\% del total de la varianza. Presenta correlaciones positivas con las siguientes afirmaciones: "Me gusta disfrutar de la buena mesa" $(0,832)$, "Consumo diariamente frutas y verduras" 0,659 , "Desarrollo algunas actividades culturales frecuentemente" $(0,575)$ y "Salgo a cenar fuera de casa todas las semanas" $(0,309)$. Este factor fue nombrado estilo de vida "de Cultura y buena mesa", pues se asocia a actividades culturales y gastronómicas.

\section{SEGMENTACIÓN DE MERCADO}

Para el siguiente análisis se identificó, mediante análisis de conglomerados jerárquicos (método de Ward), una variable de segmentación a partir de los estilos de vida de consumidores chilenos de vino orgánico (Cuadro 1). En este sentido, las diferencias se encontraron dadas por el "estilo de vida sociable y lúdico" y el "estilo de vida de cultura y buena mesa". Cabe destacar que el estilo de vida "dinámico y natural" no determinó diferencias significativas, por cuanto no es posible asociar este atributo con algún segmento en particular. Resultados similares fueron encontrados en un estudio del mercado de vino orgánico en Grecia, en donde Tsintarakis et al. (2003) señalan que el atributo "saludable" asociado al vino orgánico en comparación con el vino convencional no determina una motivación de compra con diferencia satisfactoria.

En el caso de las variables descriptivas de consumo, las diferencias estuvieron dadas por la "frecuencia de consumo de vino" y por la "cantidad de vino consumida en cada ocasión". En otro aspecto, las actitudes que otorgaron diferencias estuvieron dadas por "consume vino orgánico porque es amigable con el medio ambiente", "consume vino orgánico porque su sabor es diferente" y "consume vino orgánico porque prefiere los productos orgánicos”. Finalmente, las variables sociodemográficas que presentaron diferencias estuvieron dadas por "nivel de conocimiento sobre vino", "género" y "renta familiar mensual". Resultados similares fueron obtenidos por Fraj et al. (2004), quienes determinaron que variables psicográficas como valores, estilos de vida, personalidad y actitudes son determinantes claves en el comportamiento del consumidor de productos orgánicos.

De acuerdo a lo anterior, se identificaron tres segmentos denominados "Sociables e indiferentes hacia los productos orgánicos", los cuales reúnen el 40,32\% de los encuestados, "Actitud positiva hacia el vino orgánico", representando al 22,58\% de los encuestados, y "Consumidores de productos orgánicos" que correspondieron al 37,10\% restante.

Segmento 1. Sociables e indiferentes hacia el vino orgánico $(40,32 \%)$. El estilo de vida de los consumidores de este segmento fue denominado "sociable y lúdico", pues destinan su tiempo libre a viajar y a reunirse con amigos (Cuadro 1). La frecuencia de consumo de vino predominante en este segmento es "ocasional" $(44,0 \%)$ aun cuando la intensidad de consumo (cantidad de vino consumida en cada ocasión) es de "entre una a tres copas" (61,5\%), mientras que un $38,5 \%$ de este segmento consume "más de una botella". En relación a las actitudes de este segmento hacia el vino orgánico, los consumidores de este segmento presentan respuestas negativas hacia las tres actitudes que marcan diferencias significativas entre los segmentos, lo que determina que este grupo de consumidores sea denominado como "indiferentes hacia el vino orgánico". En relación a las variables sociodemográficas que describen a este segmento, se aprecia que son consumidores con un nivel "medio" de conocimiento de vino $(61,5 \%)$, preferentemente "hombres" $(85,7 \%)$, con una renta familiar mensual ubicada en los segmentos más bajos, predominando el "menos de 450 mil pesos" $(23,1 \%)$ (Cuadro 2).

Segmento 2. Actitud positiva hacia el vino orgánico $(22,58 \%)$. Estos consumidores no se inclinan hacia ningún estilo de vida definido, aunque presentan un menor rechazo hacia un estilo de vida "sociable y lúdica" (Cuadro 1). La frecuencia de consumo de vino característica de este segmento es la "mensual" ( $42,89 \%)$, descrita por una intensidad de consumo de "menos de una copa" $(35,7 \%)$. En general los consumidores de este segmento son "hombres" (85,7\%), con un nivel de conocimiento sobre el vino "bajo" $(50,0 \%)$. La renta familiar mensual de este segmento es bastante variable, predominando un grupo equivalente al $28,6 \%$ con de "entre 600 y 750 mil pesos" (Cuadro 2). 


\section{Cuadro 1}

Caracterización de segmentos de mercado para consumidores de vino orgánico en Chile, en función de sus estilos de vida (ANDEVA de un factor)

\begin{tabular}{|l|c|c|c|}
\hline $\begin{array}{c}\text { Factores asociados a } \\
\text { los estilos de vida }\end{array}$ & $\begin{array}{c}\text { Segmento 1 } \\
\text { Sociables e indiferente hacia } \\
\text { el vino orgánico } \\
\mathbf{( 4 0 , 3 2 \% )}\end{array}$ & $\begin{array}{c}\text { Segmento 2 } \\
\text { Actitud positiva hacia el } \\
\text { vino orgánico } \\
\mathbf{( 2 2 , 5 8 \% )}\end{array}$ & $\begin{array}{c}\text { Segmento 3 } \\
\text { Consumidores de productos } \\
\text { orgánicos } \\
\mathbf{( 3 7 , 1 0 \% )}\end{array}$ \\
\hline $\begin{array}{l}\text { Estilo de vida } \\
\text { sociable y lúdico* }\end{array}$ & $\begin{array}{c}0,983 \\
(1,030)\end{array}$ & $\begin{array}{c}-0,674 \\
(0,739)\end{array}$ & $-0,099$ \\
\hline $\begin{array}{l}\text { Estilo de vida de } \\
\text { cultura y buena } \\
\text { mesa* }\end{array}$ & $\begin{array}{c}0,321 \\
(0,831)\end{array}$ & $-1,233$ & $(0,843)$ \\
\hline
\end{tabular}

* Diferencias significativas al 1\%. Valores dentro del paréntesis representan la desviación estándar de cada promedio.

Segmento 3. Consumidores de productos orgánicos $(37,10 \%)$. El estilo de vida de este segmento es "cultura y buena mesa" pues son consumidores que disfrutan de la buena mesa y de actividades relacionadas con la cultura (Cuadro 1). La frecuencia de consumo predominante es "semanal" $(82,6 \%)$, aun cuando la opción "diaria" $(8,7 \%)$ es también de importancia al comparar con el total de la muestra $(6,0 \%)$. La cantidad de vino consumida en cada ocasión es "entre una a tres copas", ambas con un 56,5\% del total del segmento, mientras que las opciones "menos de una copa" y "entre media y una botella" también presentan importancia al compararlas con el total de la muestra. Los consumidores de este segmento declararon que consumían vino orgánico porque "prefieren los productos orgánicos" $(56,5 \%)$, demostrando una actitud positiva hacia los productos orgánicos, lo que determina que a este conjunto de consumidores se les denomine como los "consumidores orgánicos". Dichos consumidores son en su mayoría "mujeres" $(56,5 \%)$ con un conocimiento sobre el vino "medio" $(73,9 \%)$ y "alto" $(26,1 \%)$. El ingreso familiar mensual de este segmento fluctúa en los rangos de mayor poder adquisitivo, presentando un $39,1 \%$ de este segmento ingresos de "entre 750 y 900 mil pesos", seguido por un $34,8 \%$ de ingresos de "más de 900 mil pesos" (Cuadro 2). Particularmente, este segmento se vincula claramente a los resultados obtenidos por Fraj et al. (2004), quienes demostraron la hipótesis de que las personas que se caracterizan por un menor grado de extroversión y vanidad, y un mayor nivel de imaginación e intelecto, mostrarían una mayor propensión a preferir productos orgánicos.

\section{ATRIBUTOS VALORADOS POR CONSUMIDORES DE VINO ORGÁNICO}

En relación al análisis conjunto desarrollado (Cuadro 3), se identifica el perfil "ideal" del producto con arreglo a los atributos evaluados. En este caso, los consumidores manifiestan que su intención de compra se ve más afectada por la "variedad vinificada" (28,53\%), seguida por la "denominación de origen" $(27,01 \%)$, "viña productora" $(23,77 \%)$ y "precio" $(20,69 \%)$. En este sentido el producto preferido sería vino orgánico de variedad Cabernet sauvignon con precio de 2.500 pesos por botella (750 cc), producida por Concha y Toro bajo la denominación de origen Maipo. Es importante destacar que los consumidores entrevistados, además de dar una baja importancia relativa hacia el atributo "precio", se inclinan por un precio medio, en el tramo de precios considerado, lo cual puede significar que estarían dispuestos a pagar precios más elevados por este tipo de producto.

Resultados similares han sido determinados por autores que han estudiado las preferencias hacia el vino en general. Tal es el caso de Lockshin et al. (2006), quienes determinaron en el mercado australiano que existen atributos del vino que se encuentran altamente relacionados con la intención de compra de los consumidores, dentro de los que destacan la marca, región de producción (denominación de origen), precio y premios obtenidos.

Existen también algunas similitudes con investigaciones realizadas en el mercado griego. En el mismo sentido, Tzimitra-Kalogianni et al. 


\section{Cuadro 2}

Caracterización de segmentos de mercado para consumidores de vino orgánico en Chile, en función de variables descriptivas de consumo y actitudes hacia el vino orgánico. (Prueba de Hipótesis Chi-cuadrado)

\begin{tabular}{|c|c|c|c|c|}
\hline $\begin{array}{l}\text { Variables descriptivas } \\
\text { de consumo }\end{array}$ & $\begin{array}{c}\text { Segmento } 1 \\
\text { Sociables e indiferente } \\
\text { hacia el vino orgánico } \\
(\mathbf{4 0 , 3 2 \% )}\end{array}$ & $\begin{array}{c}\text { Segmento } 2 \\
\text { Actitud positiva hacia el } \\
\text { vino orgánico } \\
(\mathbf{2 2 , 5 8 \% )}\end{array}$ & $\begin{array}{c}\text { Segmento } 3 \\
\text { Consumidores de } \\
\text { productos orgánicos } \\
(37,10 \%)\end{array}$ & $\begin{array}{c}\text { TOTAL } \\
\text { MUESTRA } \\
(100,00 \%)\end{array}$ \\
\hline \multicolumn{5}{|c|}{ Frecuencia de consumo de vino** } \\
\hline Ocasional & $\begin{array}{c}7 \\
(53,8 \%)\end{array}$ & $\begin{array}{c}3 \\
(21,4 \%)\end{array}$ & $\begin{array}{c}2 \\
(8,7 \%)\end{array}$ & $\begin{array}{c}12 \\
(24,0 \%)\end{array}$ \\
\hline Mensual & $\begin{array}{c}1 \\
(7,7 \%)\end{array}$ & $\begin{array}{c}6 \\
(42,9 \%)\end{array}$ & $\begin{array}{c}0 \\
(0,0 \%)\end{array}$ & $\begin{array}{c}7 \\
(14,0 \%)\end{array}$ \\
\hline Semanal & $\begin{array}{c}5 \\
(38,5 \%)\end{array}$ & $\begin{array}{c}4 \\
(28,6 \%)\end{array}$ & $\begin{array}{c}19 \\
(82,6 \%)\end{array}$ & $\begin{array}{c}28 \\
(56,0 \%)\end{array}$ \\
\hline Diaria & $\begin{array}{c}0 \\
(0,0 \%)\end{array}$ & $\begin{array}{c}1 \\
(7,1 \%)\end{array}$ & $\begin{array}{c}2 \\
(8,7 \%)\end{array}$ & $\begin{array}{c}3 \\
(6,0 \%)\end{array}$ \\
\hline \multicolumn{5}{|c|}{ Cantidad de vino que consume en cada ocasión* } \\
\hline Menos de 1 copa & $\begin{array}{c}0 \\
(0,0 \%)\end{array}$ & $\begin{array}{c}5 \\
(35,7 \%)\end{array}$ & $\begin{array}{c}6 \\
(26,1 \%)\end{array}$ & $\begin{array}{c}11 \\
(22,0 \%)\end{array}$ \\
\hline Entre 1 a 3 copas & $\begin{array}{c}8 \\
(61,5 \%)\end{array}$ & $\begin{array}{c}6 \\
(42,9 \%)\end{array}$ & $\begin{array}{c}13 \\
(56,5 \%)\end{array}$ & $\begin{array}{c}27 \\
(54,0 \%)\end{array}$ \\
\hline Entre $1 / 2$ a 1 botella & $\begin{array}{c}0 \\
(0,0 \%)\end{array}$ & $\begin{array}{c}2 \\
(14,3 \%)\end{array}$ & $\begin{array}{c}4 \\
(17,4 \%)\end{array}$ & $\begin{array}{c}6 \\
(12,0 \%)\end{array}$ \\
\hline Más de 1 botella & $\begin{array}{c}5 \\
(38,5 \%)\end{array}$ & $\begin{array}{c}1 \\
(7,1 \%)\end{array}$ & $\begin{array}{c}0 \\
(0,0 \%)\end{array}$ & $\begin{array}{c}6 \\
(12,0 \%)\end{array}$ \\
\hline
\end{tabular}

\begin{tabular}{|c|c|c|c|c|}
\hline $\begin{array}{l}\text { Actitudes hacia } \\
\text { el vino orgánico }\end{array}$ & $\begin{array}{c}\text { Segmento } 1 \\
\text { Sociables e indiferente } \\
\text { hacia el vino orgánico } \\
\qquad(40,32 \%)\end{array}$ & $\begin{array}{c}\text { Segmento } 2 \\
\text { Actitud positiva hacia el } \\
\text { vino orgánico } \\
(\mathbf{2 2 , 5 8 \% )}\end{array}$ & $\begin{array}{c}\text { Segmento } 3 \\
\text { Consumidores de } \\
\text { productos orgánicos } \\
(37,10 \%)\end{array}$ & $\begin{array}{c}\text { TOTAL } \\
\text { MUESTRA } \\
(100,00 \%)\end{array}$ \\
\hline \multicolumn{5}{|c|}{ Consume vino orgánico porque es amigable con el medio ambiente* } \\
\hline No & $\begin{array}{c}10 \\
(76,9 \%)\end{array}$ & $\begin{array}{c}1 \\
(7,1 \%)\end{array}$ & $\begin{array}{c}9 \\
(39,1 \%)\end{array}$ & $\begin{array}{c}20 \\
(40,0 \%)\end{array}$ \\
\hline Sí & $\begin{array}{c}3 \\
(23,1 \%)\end{array}$ & $\begin{array}{c}13 \\
(92,9 \%)\end{array}$ & $\begin{array}{c}14 \\
(60,9 \%)\end{array}$ & $\begin{array}{c}30 \\
(60,0 \%)\end{array}$ \\
\hline \multicolumn{5}{|c|}{ Consume vino orgánico porque su sabor es diferente* } \\
\hline No & $\begin{array}{c}9 \\
(69,2 \%)\end{array}$ & $\begin{array}{c}7 \\
(50,0 \%)\end{array}$ & $\begin{array}{c}21 \\
(93,1 \%)\end{array}$ & $\begin{array}{c}37 \\
(74,0 \%)\end{array}$ \\
\hline Sí & $\begin{array}{c}4 \\
(30,8 \%)\end{array}$ & $\begin{array}{c}7 \\
(50,0 \%)\end{array}$ & $\begin{array}{c}2 \\
(8,7 \%)\end{array}$ & $\begin{array}{c}13 \\
(26,0 \%)\end{array}$ \\
\hline \multicolumn{5}{|c|}{ Consume vino orgánico porque prefiere los productos orgánicos* } \\
\hline No & $\begin{array}{c}12 \\
(92,3 \%)\end{array}$ & $\begin{array}{c}4 \\
(28,6 \%)\end{array}$ & $\begin{array}{c}10 \\
(43,5 \%)\end{array}$ & $\begin{array}{c}26 \\
(52,0 \%)\end{array}$ \\
\hline Sí & $\begin{array}{c}1 \\
(7,7 \%)\end{array}$ & $\begin{array}{c}10 \\
(71,4 \%)\end{array}$ & $\begin{array}{c}13 \\
(56,5 \%)\end{array}$ & $\begin{array}{c}24 \\
(48,0 \%)\end{array}$ \\
\hline
\end{tabular}




\begin{tabular}{|c|c|c|c|c|}
\hline $\begin{array}{c}\text { Variables } \\
\text { sociodemográficas }\end{array}$ & $\begin{array}{c}\text { Segmento 1 } \\
\text { Sociables e indiferente } \\
\text { hacia el vino orgánico } \\
\qquad(\mathbf{4 0 , 3 2 \% )}\end{array}$ & $\begin{array}{c}\text { Segmento } 2 \\
\text { Actitud positiva hacia el } \\
\text { vino orgánico } \\
(\mathbf{2 2 , 5 8 \% )}\end{array}$ & $\begin{array}{c}\text { Segmento 3 } \\
\text { Consumidores de } \\
\text { productos orgánicos } \\
(37,10 \%)\end{array}$ & $\begin{array}{c}\text { TOTAL } \\
\text { MUESTRA } \\
(100,00 \%)\end{array}$ \\
\hline \multicolumn{5}{|c|}{ Nivel de conocimiento sobre el vino * } \\
\hline Bajo & $\begin{array}{c}0 \\
(0,0 \%)\end{array}$ & $\begin{array}{c}7 \\
(50,0 \%)\end{array}$ & $\begin{array}{c}0 \\
(0,0 \%)\end{array}$ & $\begin{array}{c}7 \\
(14,4 \%)\end{array}$ \\
\hline Medio & $\begin{array}{c}8 \\
(61,5 \%)\end{array}$ & $\begin{array}{c}7 \\
(50,0 \%)\end{array}$ & $\begin{array}{c}17 \\
(73,9 \%)\end{array}$ & $\begin{array}{c}32 \\
(64,0 \%)\end{array}$ \\
\hline Alto & $\begin{array}{c}5 \\
(38,5 \%)\end{array}$ & $\begin{array}{c}0 \\
(0,0 \%)\end{array}$ & $\begin{array}{c}6 \\
(26,1 \%)\end{array}$ & $\begin{array}{c}11 \\
(22,0 \%)\end{array}$ \\
\hline \multicolumn{5}{|l|}{ Género*** } \\
\hline Hombre & $\begin{array}{c}11 \\
(84,6 \%)\end{array}$ & $\begin{array}{c}12 \\
(85,7 \%)\end{array}$ & $\begin{array}{c}10 \\
(43,5 \%)\end{array}$ & $\begin{array}{c}33 \\
(66,0 \%)\end{array}$ \\
\hline Mujer & $\begin{array}{c}2 \\
(15,4 \%)\end{array}$ & $\begin{array}{c}2 \\
(14,3 \%)\end{array}$ & $\begin{array}{c}13 \\
(56,5 \%)\end{array}$ & $\begin{array}{c}17 \\
(34,0 \%)\end{array}$ \\
\hline \multicolumn{5}{|l|}{ Renta Mensual Familiar*** } \\
\hline Menos de 450 mil pesos & $\begin{array}{c}3 \\
(23,1 \%)\end{array}$ & $\begin{array}{c}2 \\
(14,3 \%)\end{array}$ & $\begin{array}{c}0 \\
(0,0 \%)\end{array}$ & $\begin{array}{c}5 \\
(10,0 \%)\end{array}$ \\
\hline Entre 450 a 600 mil pesos & $\begin{array}{c}5 \\
(38,5 \%)\end{array}$ & $\begin{array}{c}4 \\
(28,6 \%)\end{array}$ & $\begin{array}{c}0 \\
(0,0 \%)\end{array}$ & $\begin{array}{c}9 \\
(18,0 \%)\end{array}$ \\
\hline Entre 600 a 750 mil pesos & $\begin{array}{c}5 \\
(38,5 \%)\end{array}$ & $\begin{array}{c}2 \\
(14,3 \%)\end{array}$ & $\begin{array}{c}6 \\
(26,1 \%)\end{array}$ & $\begin{array}{c}13 \\
(26,0 \%)\end{array}$ \\
\hline Entre 750 a 900 mil pesos & $\begin{array}{c}0 \\
(0,0 \%)\end{array}$ & $\begin{array}{c}3 \\
(21,4 \%)\end{array}$ & $\begin{array}{c}9 \\
(39,1 \%)\end{array}$ & $\begin{array}{c}12 \\
(24,0 \%)\end{array}$ \\
\hline Más de 900 mil pesos & $\begin{array}{c}0 \\
(0,0 \%)\end{array}$ & $\begin{array}{c}3 \\
(21,4 \%)\end{array}$ & $\begin{array}{c}8 \\
(34,8 \%)\end{array}$ & $\begin{array}{c}11 \\
(22,0 \%)\end{array}$ \\
\hline
\end{tabular}

* Diferencias significativas al $1 \%$.

** Diferencias significativas al $5 \%$ entre los diferentes segmentos y

*** Diferencias significativas al 10\%. Valores dentro del paréntesis representan las participaciones porcentuales dentro de cada segmento.

\section{Cuadro 3}

Preferencias de consumidores chilenos hacia vinos orgánicos de variedad Ensamble, Carmènere y Cabernet sauvignon

\begin{tabular}{|c|c|c|c|}
\hline Relativa importancia & Utilidad & Factor & Niveles \\
\hline \multirow{2}{*}{$28,53 \%$} & $(0,398)$ & Variedad vinificada & $\begin{array}{c}\text { Ensamble } \\
\text { Carmènere } \\
\text { Cabernet sauvignon }\end{array}$ \\
\hline \multirow{2}{*}{$20,69 \%$} & 0,131 & & 2.250 \\
& 0,268 & Precio & 2.500 \\
(pesos chilenos) & 2.750 \\
\hline $23,77 \%$ & 0,180 & Viña productora & Concha y Toro \\
& $(0,038)$ & Ventisquero \\
\hline \multirow{2}{*}{$27,01 \%$} & 0,451 & Denominación de origen & Maule \\
& $(0,451)$ & & Maipú \\
\hline $\mathrm{B}=1,35$ & $(0,087)$ & Niv. Significación. $=0,00001$ \\
\hline Constante $=5,9208$ & Kendall's tau $=0,944$ & \multicolumn{2}{|c|}{ Niv. Significación. $=0,0002$} \\
\hline
\end{tabular}


(1999) señalan que los atributos más valorados por consumidores griegos de vino son intrínsecos, dentro de los que destacan el sabor, aroma y claridad, como también atributos extrínsecos como denominación de origen y diseño de la etiqueta.

Finalmente, los resultados formulados en esta investigación también marcan similitudes con los obtenidos en el mercado español de vino. MartínezCarrasco et al. (2006) señalan que existen atributos del vino que influencian significativamente la decisión de compra, los que varían en importancia dependiendo de la frecuencia de consumo. Es así como los segmentos de consumidores esporádicos y consumidores ocasionales valoran en mayor medida la denominación de origen, secundado por otros atributos como tipo de vino, precio y ocasión, mientras que el segmento de consumidores habituales valoran en mayor medida el tipo de vino (joven, crianza o reserva), secundado por la denominación de origen, precio y ocasión.

\section{LITERATURA CITADA}

BARROSO, M.; BRIZ, J.; GRANDE, I. 2004. Estructura de las preferencias de los consumidores y segmentación del mercado, respecto al vino verde del norte de Portugal. Comunicación presentada en V Congreso de Economía Agraria. Santiago de Compostela, 15-17 de septiembre de 2004

BERNABÉU, R.; TENDERO, A.; OLMEDA, M.; CASTILLO, S. 2001. Actitud del consumidor de vino con Denominación de Origen en la provincia de Albacete. Comunicación presentada al IV Congreso de Economía Agraria. Pamplona. Septiembre 2001.

BERNABÉU, R.; DÍAZ, M.; OLMEDA, M.; CASTILLO, S. 2004. Análisis de segmentación de mercado de los consumidores de Vino en Castilla-La Mancha. Comunicación presentada en V Congreso de Economía Agraria. Santiago de Compostela, 15-17 de septiembre de 2004.

BIGNÉ, J. E. 1997. El consumidor verde: Bases de un modelo de comportamiento. ESIC MARKET. Abril-Junio. pp. 29-34.

BRUGAROLAS, M.; MARTÍNEZ-CARRASCO, L.; MARTÍNEZ, A.; RICO, M. 2004. Estimación de la disposición a pagar por un vino ecológico mediante el método de valoración contingente. Comunicación presentada al XVI Encuentro de Profesores Universitarios de Marketing. Alicante 22, 23 y 24 septiembre de 2004.

FRAJ, E.; MARTÍNEZ, E.; MONTANER, T. 2004. La compra de productos ecológicos explicada a través de las características psicográficas del consumidor. Congreso Nacional de la Asociación Científica del Departamento de Economía y Dirección de la Empresa (ACEDE), Murcia, España.

FRAJ, E.; MARTINEZ, E. 2002. Comportamiento del consumidor ecológico. ESIC Editorial. Madrid.

GENTILE, N.; RODRÍGUEZ, E. 2005. El consumo de alimentos orgánicos: Aplicación de un modelo logit multinomial en la elección del canal de compra. Publicación del Departamento de Economía Agraria. Facultad de Cs. Económicas y Sociales. Universidad Nacional de Mar del Plata.

GIFFORD, K.; BERNARD, J. 2006. Influencing consumer purchase likelihood of organic food. International Journal of Consumers Studies. March 2006, pp. 155-163.

GIL, J.M.; SÁNCHEZ, M. 1997. "Consumer preferences for wine attributes: a conjoint approach". British Food Journal, Vol. 99, No 1, pp. 3-11.
HAIR, J.; ANDERSON, R.; TATHAN, R.; W. BLACK. 1999. Análisis multivariante. $5^{\mathrm{a}}$ Edición. Prentice Hall. Ciudad de México, México. 715 p.

HOWARD, P.; ALLEN, P. 2006. Beyond organic: consumer interest in new labelling schemes in the Central Coast of California. International Journal of Consumer Studies. Vol. 30 (5), pp. 439-451.

JOHNSON, L.W.; RINGHAM, L.; JURD, K. 1991. Behavioural segmentation in the Australian wine market using conjoint choice analysis. International Marketing Review. Vol. 8 , $\mathrm{N}^{\circ} 4$, pp. 26-31.

KAFKA, C.; VON ALVENSLEBEN, R. 1998. Willingness to pay for organic food: A comparison between survey data and panel data from Denmark. Disponible en: http:// orgprints.org/00001754

KASSARJIAN, H. H. 1971. Personaliy and consumer behavior: A review. Journal of Marketing Research: Vol. VIII. Noviembre, pp. 409-418.

KAVAK, B.; GUMUSLUOGLU, L. 2006. Segmenting food markets. The role of ethnocentrism and lifestyle in understanding purchasing intentions. International journal of Market Research. Vol. 49 (1), pp. 71-94.

LAROCHE, M.; BERGERON, J.; BARBARO-FORLEO, G. 2001. Targeting consumers who are willing to pay more for environmentally friendly products. Journal of Consumer Marketing. Vol. 18 (6), pp. 503-520.

LOCKSHIN, L.; JARVIS, W.; D'HAUTEVILLE, F.; PERROUTY, J. 2006. Using simulations from discrete choice experiments to measure consumer sensitivity to brand, region, price and awards in wine choice. Food Quality and Preference. Vol. 17, pp. 166-178.

LUQUE, T. 2000. Técnicas de análisis de datos en investigación de mercados. Ediciones Pirámide. Madrid, España. 557 p.

MALHOTRA, N. 1997. Investigación de mercado: un enfoque práctico. 2a. ed. Prentice-Hall Hispanoamérica, S.A. Ciudad de México, México. 890 p.

MARTÍNEZ -CARRASCO, L. 2002. Problemática comercial de los vinos con D. O. Alicante. Propuesta de estrategias comerciales a partir del análisis de las preferencias de los consumidores alicantinos. Tesis Doctoral. Departamento de Estudios Económicos y Financieros. Universidad Miguel Hernández de Elche.

MARTÍNEZ-CARRASCO, L.; BRUGAROLAS, M.; DEL CAMPO, F.; MARTÍNEZ, A. 2006. Influence of 
purchase place and consumption frequency over quality wine preferences. Food Quality and Preference. Vol. 17. pp. 315-327.

MORA, G. M. 2004. Estudio de las actitudes y percepciones de los consumidores hacia los vinos de las Denominaciones de Origen de la Comunidad Valenciana. Tesis Doctoral. Departamento de Estudios Económicos y Financieros. Universidad Miguel Hernández de Elche.

MORA, G. M.; ESPINOZA, J.A. (2005). Segments determination of fresh peaches' consumers through the conjoint analysis: an approximation to the Chilean market. Sixth International Peach Symposium. Peach Culture Working Group. ISHS FRUIT SECTION. Santiago (Chile), 9-14 January, 2005. Hotel Sheraton. Enviado a Acta Horticulturae.
MORA, M.; INFANTE, R.; ESPINOZA J. A.; PREDIERI, S. 2006. Actitudes y preferencias de consumidores chilenos e italianos hacia los damascos. Revista de Economía Agraria. Vol. 10. ISSN: 0717-3520.

SCHNETTLER, B.; RUIZ, D.; SEPÚLVEDA, O. 2007. Importancia del origen en el consumo de alimentos en la IX Región de Chile. IDESIA (Chile) Septiembre-Diciembre 2007. Volumen 25, No 3, pp. 19-29.

TSINTARAKIS, C.; SISKOS, Y.; GRIGOROUDIS, E.; BAOURAKIS, G. 2003. Customer satisfaction evaluation for Greek organic wine. Proceeding of the 72d EAAE Seminar, Chania, Crete, 7-10 June.

TZIMITRA-KALOGIANNI, A.; ALEXANKI, A.; TSAKIRIDOU, E. 1999. Wine routes in northern Greece: consumer perceptions. British Food Journal, 101 (11): 884-892. 
